\title{
From Synthesis and Spectral Analysis to Molecular Modeling - Multidimensional Teaching of Medicinal Chemistry: Aspirin as an Example
}

\author{
Andrzej Polski', Agnieszka A. Kaczor ${ }^{2,3}$, Karolina Sobotka-Polska ${ }^{4}$ and Monika Pitucha ${ }^{4 *}$ \\ 1Department of Applied Pharmacy, Medical University of Lublin, 1 Chodźki St., PL-20-093 Lublin, Poland. \\ ${ }^{2}$ Department of Chemical Technology of Pharmaceutical Substances with Computer Modeling Lab, Medical University of Lublin, 4a \\ Chodźki St., PL-20-093 Lublin, Poland. \\ ${ }^{3}$ Department of Pharmaceutical Chemistry, School of Pharmacy, University of Eastern Finland, Yliopistonranta 1, P.O. Box 1627, FI- \\ 70211 Kuopio, Finland. \\ ${ }^{4}$ Department of Organic Chemistry, Medical University, Lublin, 4a Chodźki St., PL-20-093 Lublin, Poland.
}

\begin{abstract}
Classical medicinal chemistry classes do not give satisfactory learning outcomes. Computing and communication technology have a great impact on all aspects of cognition, education and training. Thus, the field of computer-assisted science teaching is particularly active nowadays. Many authors reported that involving computers in education process makes it more interesting, develops motivation of the students and helps to keep their attention during classes. Using computers for teaching science is more and more common on all the levels of education, from primary to tertiary. In particular, computer-assisted classes may be of valuable help for the pharmacy or chemistry students who learn organic or medicinal chemistry. Therefore, the main objective of this study was to design modern and interesting medical chemistry computer-assisted laboratory for students. The proposed laboratory is a hybrid of both classic (synthesis of aspirin, TLC analysis, ${ }^{1} \mathrm{H},{ }^{13} \mathrm{C}$ NMR and IR spectra) and new (molecular modeling) chemistry elements. We suggest a simple and safe method for the synthesis of aspirin and its analysis with spectroscopic methods. In addition, the laboratory includes the use of computational methods for the analysis of aspirin and its mechanism of action on the molecular level. These procedures are easy to use and can be easily carried out even by inexperienced students. Moreover, the proposed laboratory can be used as an example to create similar classes with the use of other drugs, it can be easily reproduced anywhere and does not require any special, expensive computer resources, being based on free and easy available software.
\end{abstract}

Key words: Aspirin, Computer-Assisted Classes, Organic Chemistry, Molecular Modeling, Spectroscopic Method.

\section{INTRODUCTION}

Many organic chemistry laboratories for students have been published. For example, Birney and Starnes proposed simple laboratory classes involving synthesis and identification of methyl salicylate. ${ }^{1}$ Furthermore, Reed and Hutchison described classes on synthesis of adipic acid. ${ }^{2}$ The common problem with a such type of classes is that they expose students to a "do this, see that" without proper understanding of the process. ${ }^{3}$ Classical classes or laboratories, where students only listen to or do subsequent tasks without any proper understanding of the topic, do not give satisfactory learning outcomes. It is caused by the fact that students have problems with "seeing" the molecules, understanding their shapes and reactions which occurs. ${ }^{4} \mathrm{~A}$ deeper under-
Submission Date :18-12-2014 Revision Date : :16-01-2015 Accepted Date : :19-01-2015

DOI: 10.5530/ijper.49.2.1 Correspondence Address Dr.Monika Pitucha Department of Organic Chemistry,

Medical University, Lublin, 4a Chodźki St., PL-20-093 Lublin, Poland.

E-mail:monika.pitucha@ umlub.pl

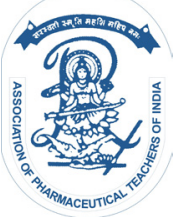

www.ijper.org 
A

\section{$\mathrm{C}_{6} \mathrm{H}_{4}\left(\mathrm{OCOCH}_{3}\right) \mathrm{COOH}$}

B

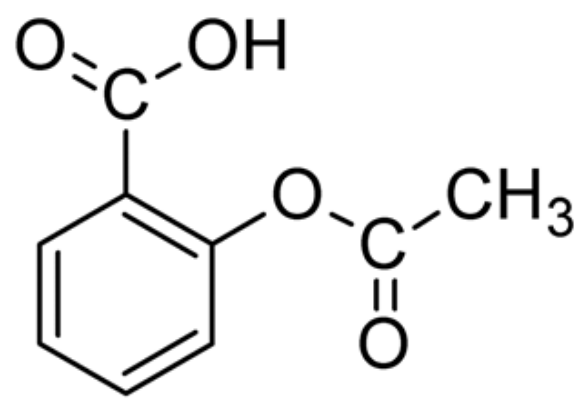

C

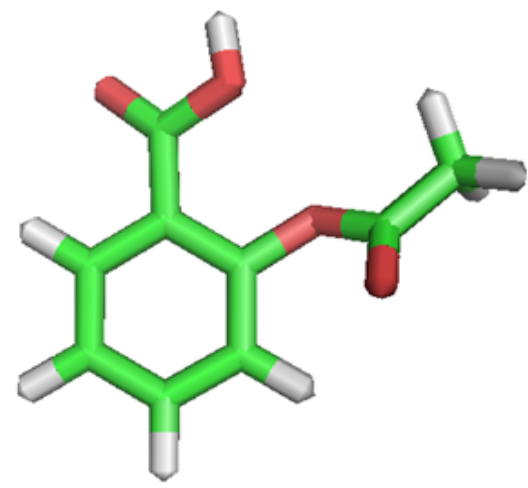

Figure 1: Evaluation of aspirin structure from 1D to 2D to 3D.

standing requires knowledge of a molecule's three dimensional shape. Figure 1 shows the same molecule of acetylsalicylic acid (aspirin), but structures differ much from each other (1-Dimension, 2-Dimensions and 3-Dimensions). The 3D representation contains more information about structure, such us: general conformation and stereochemistry, bond angles and torsion angles. Free computer software can be used by students who can draw and model chemical compounds may significantly improve the understanding of medicinal chemistry by students. ${ }^{5}$

\section{MATERIALS AND METHODS}

In organic chemistry images and symbols are used to represent different aspects of chemical phenomena. ${ }^{6}$ Molecular modeling software enables easy construction of even complicated compounds and looking for their proper geometrical structure, bond lengths and angles, and at the end observing the received model from different views. ${ }^{7}$ Computer-assisted classes allows students to be active in the learning process, shows alternative solutions of the problems and develops not only their manual skills, but also knowledge. ${ }^{8}$

Indian Journal of Pharmaceutical Education and Research | Vol 49 | Issue 2 | Apr-Jun, 2015
The chemistry and pharmacy students should also understand the importance of organic synthesis as a field of knowledge. They should be familiar with different aspects of planning, performing and monitoring the progress of the chemical reaction. Moreover, they are expected to have general knowledge of the currently used techniques and laboratory methods in accordance with the rules of safe handling of chemicals, and the selection and utilization of chemical waste.

TLC (thin-layer chromatography) is an important tool in not only in modern pharmacy, but also in analytical chemistry, biochemistry, food analysis, etc. It can be also used with success in practice of chemistry teaching because it is convenient, simple, cheap, environmentfriendly and can be easily used by inexperienced students. ${ }^{9}$ This method is often used for checking the purity and homogeneity of drugs in pharmaceutical analysis (impurity profiling). ${ }^{10}$ Moreover, a few chemistry experiments were published, which used IR spectroscopy as educational part of the laboratory. ${ }^{11,12}$ However, there are not many laboratories that extended learning of students from synthesis, spectroscopic and chromatography methods to molecular modeling The proposed laboratory consists of three main sections (synthesis, analysis and molecular modeling) and its scheme can be applied to any other chemical substances, not only to aspirin. For better understanding of the topic by students, it should be divided into at least two meetings. During the first laboratory the students should focus on synthesis of a desired substance and analysis of its purity by TLC. Second lab should begin with a discussion and performance of spectroscopic analysis. A the end students will work on molecular modeling of appropriate structures and mechanism of action of an earlier obtained drugs.

\section{RESULTS}

Acetylsalicylic acid (ASA) is a non-steroidal anti-inflammatory drug (NSAID), which has been widely used for over 100 years. It has anti-inflammatory and antipyretic effects and can be used to treat small to medium-intensity pain. ${ }^{13}$ ASA is also commonly used by patients, who have risk of stroke, myocardial infarction and unstable angina. Moreover, it has a positive effect on patients suffering from Alzheimer or Parkinson disease. ${ }^{14,15}$ Clinical studies have shown that aspirin has a significant impact on reducing the risk of pancreatic cancer and colorectal cancer. ${ }^{16,17}$

Although the synthesis of aspirin is well-known, ${ }^{18,19}$ we propose a simple method for the preparation and crystallization of aspirin which ensures to get pure product with a very good yield. Additionally, we would like to teach students how molecular modeling can be used for 


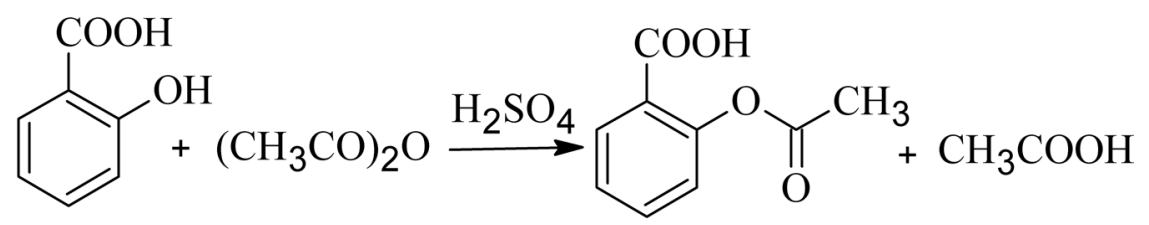

Figure 2: The synthesis of aspirin

finding drug properties and understanding its mechanism of action.

The main aim of this study was to design an interesting and easy to carry out medicinal chemistry computerassisted laboratory classes. As a model substance we chose acetylsalicylic acid. There were a few reasons for this choice. Firstly, ASA can be easily synthesized from salicylic acid and acetic acid anhydride, so reaction should be easily understood and carried out by all students. Secondly, it is a widely used NSAID, most students should know it which is expected to stimulate their curiosity how it is synthesized. Thirdly, this simple molecule is an excellent example for of the beginners in molecular modeling.

The laboratory classes consist of the following steps:

1) acetylsalicylic acid synthesis; 2) determination of obtained product purity and homogeneity using thinlayer-chromatography; 3) study of its ${ }^{1} \mathrm{H},{ }^{13} \mathrm{C} \mathrm{NMR}$ and IR spectra 4) molecular modeling.

Students perform synthesis of aspirin according to the following procedure: $11.0 \mathrm{~g}$ of salicylic acid, $15.2 \mathrm{ml}$ of acetic acid anhydride and 10 drops of concentrated sulfuric acid are placed in the round-bottom flask (capacity of $50 \mathrm{ml}$ ). The reaction mixture is heated in a water bath under Liebig condenser for half an hour at $50-60^{\circ} \mathrm{C}$ (do not exceed a temperature of $60^{\circ} \mathrm{C}$ ) under constant stirring. Upon completion of heating the content of the flask is cooled down and poured into $160 \mathrm{ml}$ of distilled water to remove excess of acetic acid anhydride. The resulting precipitate is filtered and allowed to dry. The dried precipitate is dissolved in $32 \mathrm{ml}$ of $96 \%$ ethanol, and then poured into $80 \mathrm{ml}$ of hot distilled water and left to crystallize. The product is filtered under reduced pressure and then dried at room temperature. The procedure makes it possible to obtain $12 \mathrm{~g}$ of acetylsalicylic acid (Figure 2).

Students check the purity and homogeneity of obtained product using TLC. A mixture of chloroform and methanol (ratio 10:2 with the addition of acetic acid) is used as an eluent. The spots are visualized with UV light at wavelength of 254 and $360 \mathrm{~nm}$. Later, students with the help of a teacher learn how to measure and interpret ${ }^{1} \mathrm{H},{ }^{13} \mathrm{C}$ NMR and IR spectra for aspirin.

In our case the acetylsalicylic acid (Aspirin) was obtained in $85 \%$ yield. Product was characterized by the retardation factor $(\mathrm{Rf}=0.69))$ and retention factor $(\mathrm{k}=0.449)$ and ${ }^{1} \mathrm{H},{ }^{13} \mathrm{C}$ NMR and IR spectra. NMR spectra were recorded on a $600 \mathrm{MHz}$ instrument. In the ${ }^{1} \mathrm{H}$ NMR spectrum the characteristic signals are observed: carboxyl group (13.084 ppm), aromatic proton (7.181$7.948 \mathrm{ppm}$ ) and methyl group (2.245 ppm). Carbon NMR spectrum shows characteristic signals at 169.673 ppm (carboxyl group), 166.086 (carbonyl group), 134.284-124.252 ppm (aromatic carbon) and 21.329

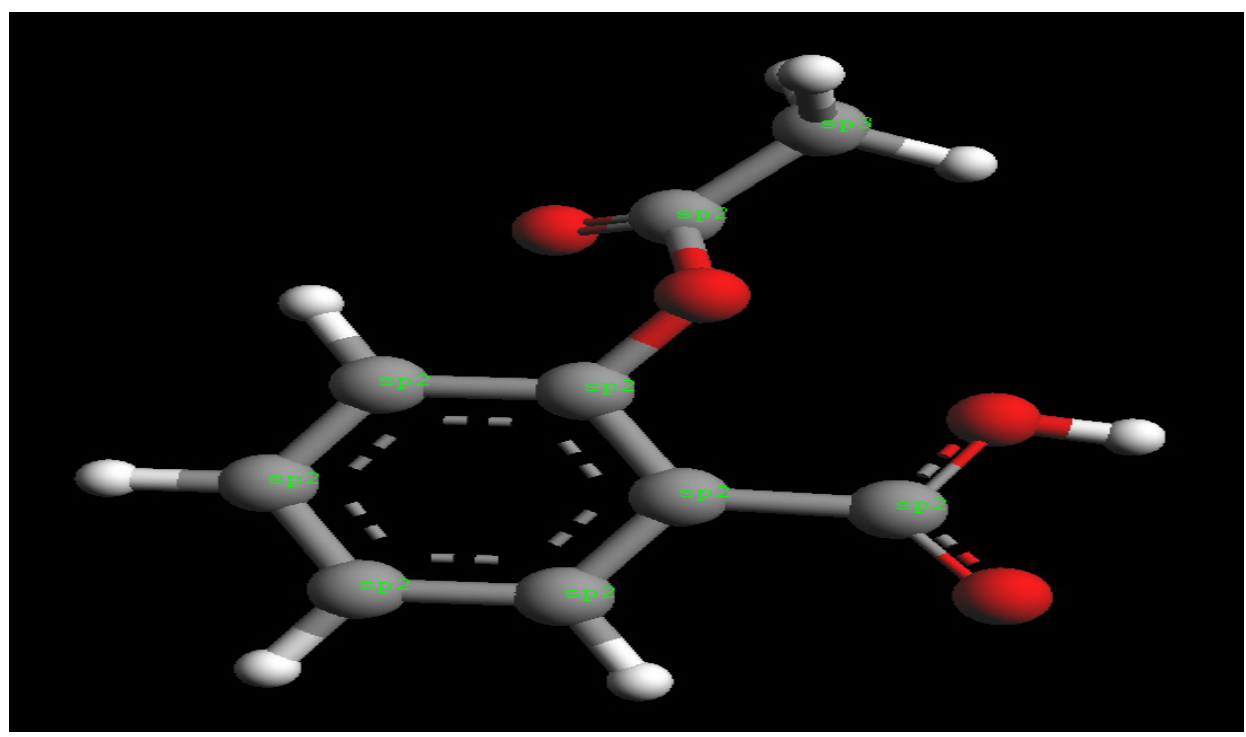

Figure 3: The hybridization of the carbon atoms in the molecule of aspirin 


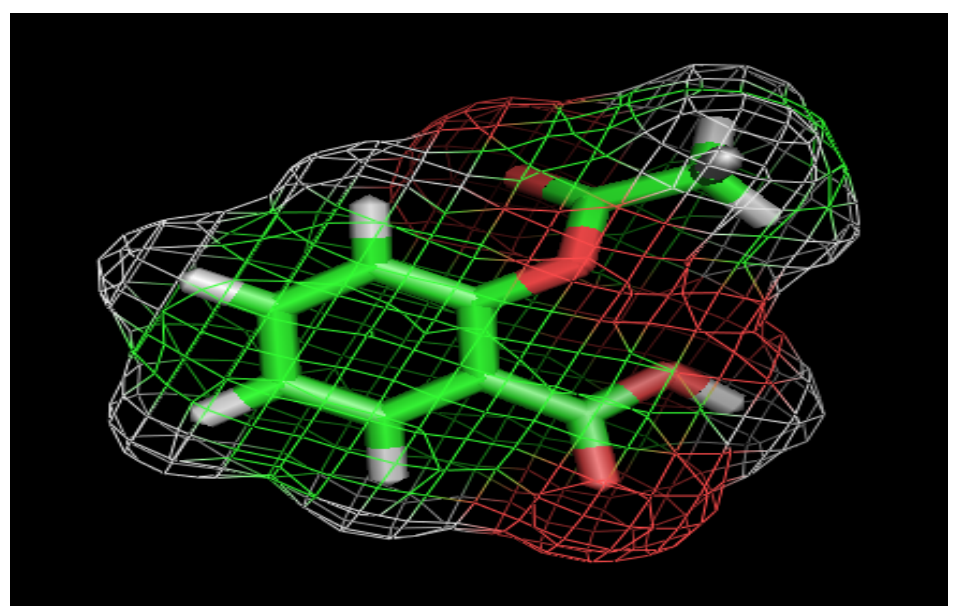

Figure 4: Molecular surface of aspirin in mesh representation

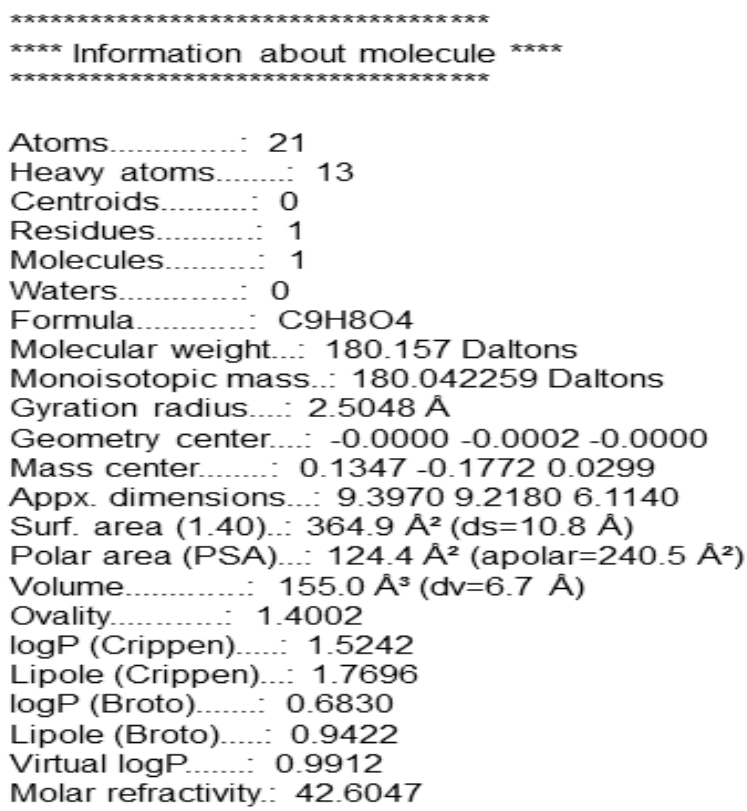

Figure 5: Molecular properties of aspirin as calculated with Vega ZZ

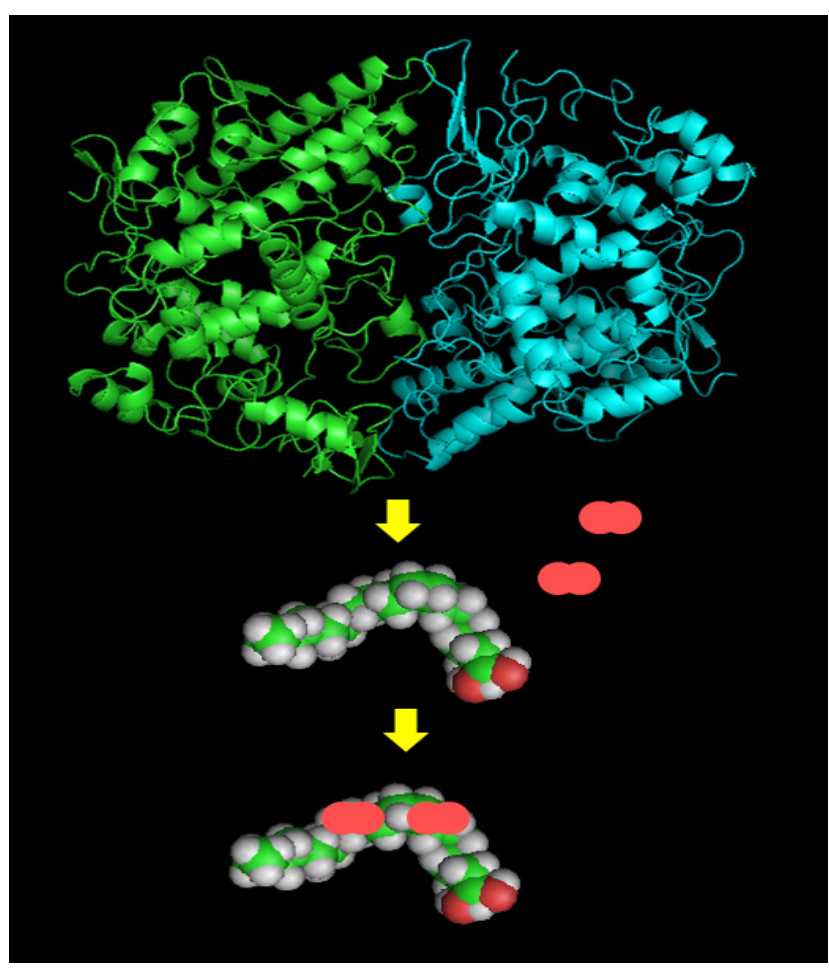

Figure 6: COX catalyzes the first step of synthesis of prostaglandins, here it adds two molecules of oxygen to the molecule of arachidonic acid

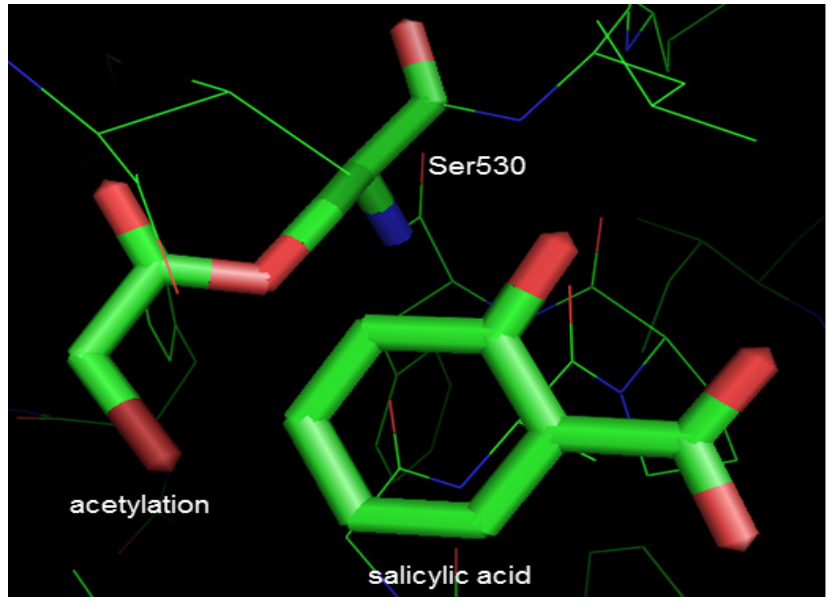

Figure 7: Aspirin blocks COX active site by acetylation of Ser530, PDB ID: 1PTH 
ppm (methyl group). IR spectra were recorded on a Thermo Nicolet 6700 FTIR spectrometer. Solids were measured utilizing the ATR technique with the spectrometer equipped with a diamond Orbit stage. In IR spectra are visible the signals in the range of 2546-2831 $\mathrm{cm}^{-1}$ confirm the presence hydroxyl group $(\mathrm{COOH})$.

The last stage of the laboratory, probably the most enjoyable for most students, is molecular modeling. The structure of aspirin is built with free Argus Lab software $^{20}$ and optimized using semiempirical AM1 method by selection from the Argus Lab main menu Calculation-Optimize geometry and marking the appropriate level of theory. Argus Lab is also used to check hybridization of the selected atoms (eg. carbon atoms) by setting atom labels from Label menu to hybridization (Figure 3). The students are reminded that the structure of carboxylic group is resonance-stabilized, which is also marked in Figure 3.

Furthermore, students are thought that the real shape of the molecule is not its skeleton but its molecular surface (Figure 4). Mesh representation of molecular surface was obtained with free software PyMol v. 99 from $S$ (how)-mesh side menu. The representation of a molecule is changed to sticks from $\mathrm{S}$ (how)-sticks side menu. Finally, free software VegaZZ is used to calculate molecular properties of aspirin from VegaZZ main menu Viev-Information-calculate (Figure 5). ${ }^{21}$

The students should note the low lipophilicity value of aspirin. They should be also able to indicate the number of hydrogen bond donors and acceptors in the molecule of aspirin and to discuss its drug-likeness according to the Lipinski rule.

The next stage of molecular modeling part of the laboratory is the discussion about features and mechanism of action of aspirin. It has analgesic, antipyretic and anti-inflammatory actions which are exerted through inhibiting the enzyme cyclooxygenase and thus preventing the formation and release of prostaglandins. ${ }^{22}$ Furthermore, aspirin slows blood clotting, reducing the chance of stroke and heart attack. Surprisingly, there is a growing body of evidence that this drug can be helpful against some types of cancer.

Cyclooxygenase (COX) is an enzyme responsible for the first step of synthesis of prostaglandins from a common fatty acid (arachidonic acid). It is a homodimeric enzyme which integrates into the single leaflet of the cell membrane. This bifunctional enzyme consists of three independent folding units: an epidermal growth factor-like domain, a membrane-binding motif and an enzymatic domain. ${ }^{22}$ The structure of an enzyme which adds two molecules of oxygen to the arachidonic acid is presented in Figure 6.
The COX active site is a long hydrophobic channel and aspirin-like drugs such as flurbiprofen inhibit COX by excluding arachidonate from the upper portion of the channel. ${ }^{22}$ Aspirin blocks the binding of arachidonic acid in the cyclooxygenase active site by acetylation of its Ser 530 (Figure 7).

\section{DISCUSSION}

The presented laboratory classes can be used as an introduction in the course of organic chemistry, medicinal chemistry or molecular modeling. It consists of both classic (synthesis of aspirin) and modern elements (usage of computer for molecular modeling) The use of computers in classes has a significant effect on the maintenance of student's attention and activity. ${ }^{23}$ Participation in such a computer-assisted laboratory will encourage students that internet can be used not only for entertainment, but also can be valuable source of easy accessible knowledge acquired in a friendly andenjoyable way.

Moreover, teaching students molecular modeling allows them to understand different techniques of visualization, manipulation and analysis of molecular structures, which are demanded for specialists working in the industry. ${ }^{24}$ The skills acquired during such as laboratory can be an useful tool for students in their future jobs.

Important advantage of this laboratory is that proposed molecular modeling uses only free software, so interested students can use the gained knowledge at home. The proposed laboratory classes can also be easily reproduced anywhere or can be a basis for further modifications and do not require any special, expensive computer resources.

\section{CONCLUSION}

We present an example of combination of experimental and computational methods in teaching medicinal chemistry. The tutorial makes it possible to familiarize students with the elements of organic synthesis and the techniques used in molecular modeling. The students learn to use spectroscopic methods for the analysis of the obtained compound. The tutorial shows how to use the freely available software for molecular modeling. The proposed experiment can be used as an individual student work or demonstration for the group of students. Fast and cheap experiment lets students acquaint with the procedures and tools in chemistry.

\section{ACKNOWLEDGEMENT}

The paper was developed using the equipment purchased within the project "The equipment of innovative laboratories doing research on new medicines used 
in the therapy of civilization and neoplastic diseases" within the Operational Program Development of Eastern Poland 2007-2013, Priority Axis I modern Economy, operations I.3 Innovation promotion. The research was partially performed during the postdoctoral fellowship of Agnieszka A. Kaczor at University of Eastern
Finland, Kuopio, Finland under Marie Curie fellowship. Part of the calculations was performed under a computational grant by Interdisciplinary Center for Mathematical and Computational Modelling (ICM), Warsaw, Poland, grant number G30-18 and under resources and licenses by CSC, Finland.

\section{REFERENCES}

1. Birney DM, Starnes SD. Parallel combinatorial Esterification. A simple experiment for use in the second-semester organic chemistry laboratory. J. Chem. Educ. 1999; 76(11): 1560-1.

2. Reed SM, Hutchison JE. Green Chemistry in the organic teaching laboratory: An environmentally benign synthesis of adipic acid. J. Chem. Educ. 2000; 77(12): 1627-9.

3. Mohrig JR. The problem with organic chemistry labs. J. Chem. Educ. 2004; 81(8): 1083-5.

4. Muzyka JL. Visualization tools for organic chemistry. J. Chem. Educ. 2009; 86(2): 254.

5. Springer T. Improving student understands of molecular structure through broad-based use of computer models in the undergraduate organic chemistry lecture. J. Chem. Educ. 2014; 91(8): 1162-8.

6. Kozma R, Russell J. Students becoming chemists: developing representational competence. In: JK. Gilbert (Ed.). Visualization in science education Dordrecht, The Netherlands: Springer; 2005. P. 121-45.

7. Barak M, Dori YJ. Enhancing undergraduate student's chemistry understanding through project-based learning environment. Sci. Educ. 2005; 89(1): 117-39.

8. Özmen $\mathrm{H}$. The influence of computer-assisted instruction on students' conceptual understanding of chemical bonding and attitude towards chemistry: A case for Turkey. Computers \& Education 2008; 51(1): 423-38.

9. Fuchs B, Süß B, Nimptsch A, Schiller J. MALDI-TOF-MS directly combined with TLC. A review of current state. Chromatographia 2009; 69(1): 95-105.

10. Ferenczi-Fodor K, Vegh Z, Renger B. Impurity profiling of pharmaceuticals by thin-layer chromatography. J. Chromatogr A. 2011; 1218(19): 2722-31.

11. Hill MA. Infrared spectroscopy in the general chemistry lab. J. Chem. Educ. 2001; 78(1): 26

12. Szalay PS. A simple experiment in the separation of a solid-phase mixture and infrared spectroscopy for introductory chemistry. J. Chem. Educ. 2008; 85(2): 285.

13. Mackowiak PA. Brief history of antipyretic therapy. Clin. Infect. Dis. 2000; 31(5): 154-6.

14. Chen $H$, Zhang SM, Hernán, MA, Schwarzschild MA, Willett WC, Colditz GA, et al. Nonsteroidal Anti-inflammatory Drugs and the Risk of Parkinson Disease. Arch. Neurol. 2003; 60(08): 1059-64.

15. Entminan M, Sudeep G, Samii A. Effect of non-steroidal anti-inflammatory drugs on risk of Alzheimer's disease: systematic review and meta-analysis of observational studies. Brit. Med. J. 2003; 327(7404): 128-30.

16. Tan $\mathrm{XL}$, Reid Lombardo $\mathrm{KM}$, Bamlet $\mathrm{WR}$, Oberg $\mathrm{AL}$, Robinson $\mathrm{DP}$ Anderson KE, et al. Aspirin, nonsteroidal anti-inflammatory drugs (NSAID), acetaminophen, and pancreatic cancer risk: A clinic-based case-control study. Cancer Prev. Res. 2011; 4(11): 1835-41.

17. Chan AT, Arber N, Burn J, Chia WK, Elwood P, Hull MA, et al. Aspirin in the Chemoprevention of Colorectal Neoplasia: An Overview. Cancer Prev. Res. 2012; 5(2): 164-78.

18. Olmsted J. Synthesis of Aspirin: A General Chemistry Experiment. J. Chem. Educ. 1998; 75(10): 1261-2

19. Byrd H, O'Donnell SE. A General Chemistry Laboratory Theme: Spectroscopic Analysis of Aspirin. J. Chem. Educ. 2003; 80(2): 1174-6.

20. Argus Lab, http://www.arguslab.com/arguslab.com/ArgusLab.html, (accessed June 2014)

21. Pedretti A, Villa L, Vistoli G. VEGA - An open platform to develop chemo-bioinformatics applications, using plug-in architecture and script programming. J. Comput. Aid. Mol. Des. 2004; 18(3): 167-73.

22. Botting RM. Vane's discovery of the mechanism of action of aspirin changed our understanding of its clinical pharmacology. Pharmacol. Rep. 2010; 62(3): 518-25.

23. Tsai CS. Micro computers applications in biochemistry. J. Chem. Educ. 2000; 77(2): 219-21.

24. Kaczor AA. Computer-assisted classes on medicinal chemistry of neuroleptics. Problems of Education in the $21^{\text {st }}$ Century $2011 ; 28: 49$. 